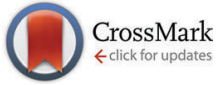

Cite this: J. Mater. Chem. C, 2016 , 4, 9036

Received 2nd August 2016, Accepted 8th September 2016

DOI: $10.1039 / c 6 t c 03319 a$

www.rsc.org/MaterialsC

\section{Towards a metallic top contact electrode in molecular electronic devices exhibiting a large surface coverage by photoreduction of silver cations}

\author{
Santiago Martín, ${ }^{\text {ab }}$ Luz M. Ballesteros, ${ }^{\text {ac }}$ Alejandro González-Orive, ${ }^{\text {acd }}$ Hugo Oliva, ${ }^{\text {ac }}$ \\ Santiago Marqués-González, ${ }^{\text {ef }}$ Matteo Lorenzoni, ${ }^{9}$ Richard J. Nichols, ${ }^{\text {h }}$ \\ Francesc Pérez-Murano, ${ }^{9}$ Paul J. Low ${ }^{\text {ei }}$ and Pilar Cea*acd
}

\begin{abstract}
In this contribution the photoreduction of silver ions coordinated onto a Langmuir-Blodgett monolayer is presented as an effective method for the deposition of the top contact electrode in metal/monolayer/ metal devices. Silver cations were incorporated from an aqueous $\mathrm{AgNO}_{3}$ sub-phase of Langmuir films of 4,4'-(1,4-phenylenebis(ethyne-2,1-diyl))dibenzoic acid upon the transference of these films onto a metallic substrate. Subsequent irradiation of the silver-ion functionalized Langmuir-Blodgett films with $254 \mathrm{~nm}$ light results in the photoreduction of silver cations to produce metallic silver nanoparticles, which are distributed over the organic monolayer and exhibit a surface coverage as large as $76 \%$ of the monolayer surface. Electrical properties of these metal/monolayer/metal devices were determined by recording $1-V$ curves, which show a sigmoidal behaviour indicative of well-behaved junctions free of metallic filaments and short-circuits. The integrity of the organic monolayer upon the irradiation process and formation of the silver top-contact electrode has also been demonstrated through cyclic voltammetry experiments.
\end{abstract}

\section{Introduction}

Despite the enormous progress in the field of molecular electronics in recent years ${ }^{1}$ many scientific and technological challenges must still be addressed before molecular electronics can be considered a mature technology capable of reaching the market. ${ }^{2}$ Whilst the assembly of a well-ordered monolayer film of electrically functional molecules on a conducting substrate

\footnotetext{
${ }^{a}$ Departamento de Química Física, Facultad de Ciencias, Universidad de Zaragoza, 50009, Spain. E-mail: pilarcea@unizar.es

${ }^{b}$ Instituto de Ciencias de Materiales de Aragón (ICMA), Universidad de Zaragoza-CSIC, 50009 Zaragoza, Spain

'Instituto de Nanociencia de Aragón (INA) y Fundación INA, edificio i+d Campus Rio Ebro, Universidad de Zaragoza, C/Mariano Esquillor, $s / n$,

50018 Zaragoza, Spain

${ }^{d}$ Laboratorio de Microscopias Avanzadas (LMA), Universidad de Zaragoza, 50018 Zaragoza, Spain

${ }^{e}$ Department of Chemistry, University of Durham, Durham DH1 3LE, UK

${ }^{f}$ Department of Chemistry, Graduate School of Science and Engineering, Tokyo Institute of Technology, Tokyo 152-8511, Japan

${ }^{g}$ Instituto de Microelectrónica de Barcelona (IMB-CNM, CSIC), Campus UAB, 08193 Bellaterra, Spain

${ }^{h}$ Department of Chemistry, University of Liverpool, Crown Street, Liverpool, L69 7ZD, UK

${ }^{i}$ School of Chemistry and Biochemistry, University of Western Australia, 35 Stirling Highway, Crawley, Perth, 6009, Australia
}

can be readily achieved by self-assembly or Langmuir-Blodgett methods, difficult challenges persist with regard to the deposition a 'top-contact' electrode onto such structures to complete the device-like structure. Significant problems in the fabrication of the top-contact electrode include damage of the functional single layer films during the deposition of the top, usually metallic, electrode by methods such as thermal evaporation, and penetration of the growing top-contact through the monolayer, which results in short circuits. Some recent reviews have analysed in detail the top-contact electrode problem, and summarised the contemporary strategies aimed at overcoming this issue. ${ }^{3-8}$

Strategies from our group concerning the fabrication of the topcontact electrode have included the thermal induced decomposition of an organometallic compound (TIDOC) method, ${ }^{9}$ chemisorption of gold nanoparticles onto a monolayer surface-functionalised with a terminal alkyne moiety $(-\mathrm{C} \equiv \mathrm{CH})$ resulting in the formation of a $\sigma$ $\mathrm{C}-\mathrm{Au}$ bond, ${ }^{10}$ and photoreduction of a gold precursor incorporated into the monolayer. ${ }^{11}$ In the latter method, a metal precursor $\left(\left[\mathrm{AuCl}_{4}\right]^{-}\right)$was incorporated onto a Langmuir-Blodgett (LB) film from the sub-phase during the fabrication process, with subsequent photoreduction leading to the formation of metallic gold nano-islands (GNIs) on top of the intact molecular film. This method required only optical illumination over the substrate area, and yielded metal|molecule|GNIs systems free of metallic inter-penetration and short circuits providing a route to 
nascent device structures. However, whilst excellent electrical contact between the underlying monolayer and the GNI-based top-contacts was achieved, the surface coverage of these GNIbased top-electrodes was sparse, and despite the extremely useful electrical properties of gold, the mobile nature of this metal prevents its use in modern electronic devices. In addition, although gold remains the work-horse material for electrodes used in molecular electronics, there is a rapidly growing body of work which has demonstrated the additional fundamental science concerning charge transport and tunnel barriers that can be gleaned from comparative studies of devices constructed from different electrode materials.

In this contribution, the soft photochemical procedure is extended to the fabrication of silver top-contacts, with a larger surface coverage of metal nanoparticles than previously achieved with gold, on monolayers of an oligo(phenylene ethynylene) (OPE) derivative, 4,4'-(1,4-phenylenebis(ethyne-2,1-diyl))dibenzoic acid $\left(\mathbf{1 H}_{2}\right.$, Fig. 1).

Compound $\mathbf{1} \mathbf{H}_{\mathbf{2}}$ is a symmetric OPE derivative, which has been shown to form homogeneous and highly ordered LangmuirBlodgett (LB) films. ${ }^{12}$ On the one hand, the proton associated with the carboxylic acid $(-\mathrm{COOH})$ of $\mathbf{1 H}_{\mathbf{2}}$ within the aqueous sub-phase is readily exchanged for other cations introduced into the aqueous sub-phase. If the majority cation in the subphase is $\mathrm{Ag}^{+}$, then a Langmuir film denoted as $\mathbf{1} \mathbf{H A g}{ }^{+}$is formed. These silver cations are transferred onto LB films to maintain the electroneutrality of the system. Silver cations also incorporate some water molecules as part of their hydration sphere. ${ }^{13}$ On the other hand, the tendency of carboxylic groups to chemisorb onto metals such as gold or silver is also well-known, ${ }^{14,15}$ and when the gold substrate is introduced in the water subphase the carboxylate group is chemisorbed onto the metal surface, which involves deprotonation of the terminal carboxylic acid to form $\mathbf{1 A g}^{+}$LB films. Subsequent photoreduction of the coordinated silver cations in $\mathbf{1 A g}^{+}$LB films results in the formation of disklike metallic silver nanoparticles, which cover a significant portion of the film surface, and this system is denoted here as 1AgNP. The photoreduction mechanism of silver cations ${ }^{16}$ as well as the subsequent nucleation and growth mechanism of the silver nanoparticles ${ }^{17,18}$ have been studied in detail before. Fig. 1 summarizes the method proposed in this work for the fabrication of metal/monolayer/metal devices.

\section{Experimental}

The compound 4,4'-[1,4-phenylenebis(ethyne-2,1-diyl)]-dibenzoic acid $\left(\mathbf{1 H}_{\mathbf{2}}\right)$ was prepared as described elsewhere. ${ }^{11}$ A Nima Teflon trough with dimensions $(720 \times 100) \mathrm{mm}^{2}$ housed in a constant temperature $\left(20 \pm 1{ }^{\circ} \mathrm{C}\right)$ clean room was used to prepare the films. The surface pressure $(\pi)$ of the monolayers was measured by using a Wilhelmy paper plate pressure sensor. Ultrapure Millipore Milli- ${ }^{\circledR}$ water (resistivity $18.2 \mathrm{M} \Omega \mathrm{cm}$ ) was used as sub-phase. The spreading solutions with a concentration of $10^{-5} \mathbf{M} \mathbf{1} \mathbf{H}_{2}$ were prepared in chloroform (HPLC grade, 99.9\% purchased from Sigma and used as received). To construct the Langmuir films, the solution was spread drop-by-drop using a Hamilton micro-syringe held very close to an aqueous surface, allowing the surface pressure to return to a value as close as possible to zero between each addition. The spreading solvent was allowed to completely evaporate over a period of at least 15 min before compression of the Langmuir film at a constant sweeping speed of $0.02 \mathrm{~nm}^{2}$ molecule ${ }^{-1} \mathrm{~min}^{-1}$. The $\Delta V-A$ measurements were carried out using a Kelvin probe provided by Nanofilm Technologie GmbH, Göttingen, Germany. The direct visualization of the monolayer formation at the air/water
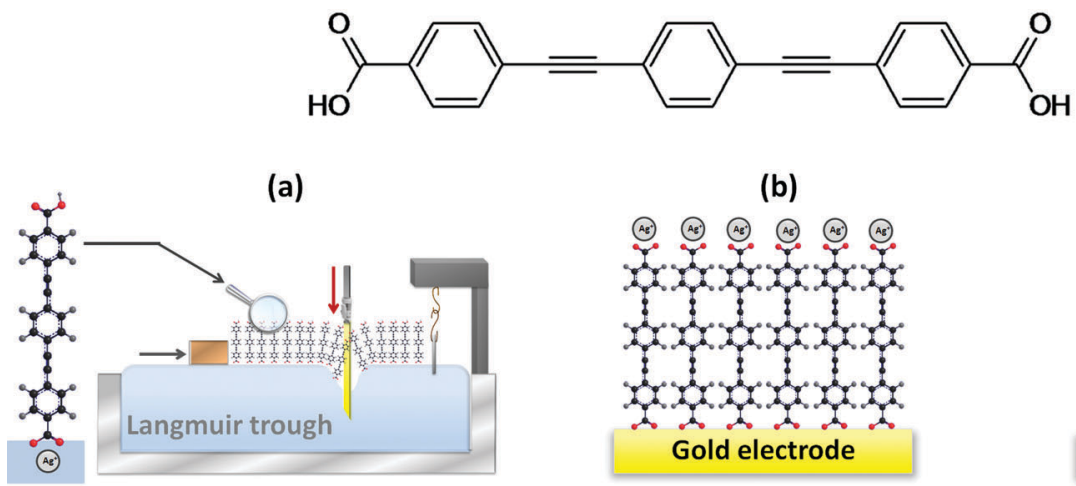

$\mathbf{1 H A g}^{+}$Langmuir monolayer (b)

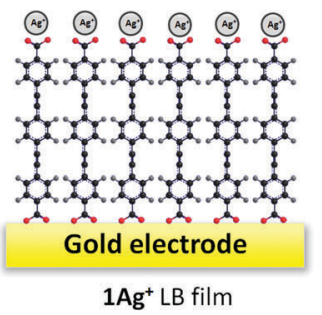

$1 \mathrm{H}_{2}$

(c)

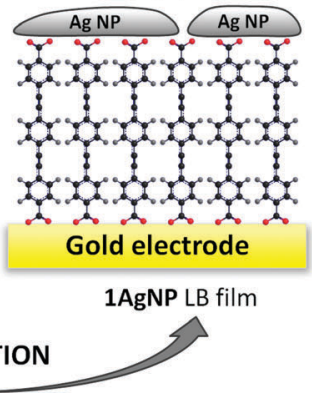

Fig. 1 Top image: 4,4'-(1,4-Phenylenebis(ethyne-2,1-diyl))dibenzoic acid $\left(\mathbf{1}_{\mathbf{2}}\right)$. Bottom image: Schematic of the Au|monolayer|Ag device fabrication strategy: (a) Langmuir film of $\mathbf{1 H}_{\mathbf{2}}$ spread onto an aqueous sub-phase containing $\mathrm{AgNO}_{3}$; the carboxylic acid in contact with the aqueous sub-phase is deprotonated and a double ionic layer incorporating the majority cation is formed, $\mathbf{1} \mathbf{H A g}^{+}$film. (b) Transference of the $1 \mathbf{H A g} \mathbf{~}^{+}$Langmuir film by immersion of a gold substrate into the water sub-phase results in the formation of a Langmuir-Blodgett (LB) monolayer in which the carboxylic group not immersed in the aqueous sub-phase is chemisorbed onto the gold substrate and the carboxylate group immersed in the aqueous subphase incorporates silver cations to maintain the electroneutrality of the system, $\mathbf{1} \mathbf{A g}^{+}$film. (c) Irradiation of the $1 \mathbf{A g}^{+}$LB monolayer results in photoreduction of the silver cations and formation of silver nanoparticles. The film is denoted as 1AgNPs. 
interface was studied using a commercial micro-Brewster angle microscopy (micro-BAM) from KSV-NIMA, having a lateral resolution better than $12 \mu \mathrm{m}$.

The films were deposited on solid substrates of quartz, mica, glass or gold depending on the characterization technique to be subsequently used, at a constant surface pressure by the vertical dipping method (substrates initially outside of the water sub-phase) with a dipping speed of $0.6 \mathrm{~cm} \mathrm{~min}^{-1}$. Gold substrates were purchased from Arrandee ${ }^{\circledR}$, Schroeer, Germany and were flame-annealed at approximately $800-1000{ }^{\circ} \mathrm{C}$ with a Bunsen burner immediately prior to use to prepare atomically flat $\mathrm{Au}(111)$ terraces. ${ }^{19}$ X-ray photoelectron spectroscopy (XPS) spectra were acquired on a Kratos AXIS ultra DLD spectrometer with a monochromatic Al K $\alpha$ X-ray source (1486.6 eV) using a pass energy of $20 \mathrm{eV}$. To provide a precise energy calibration, the XPS binding energies were referenced to the $\mathrm{C} 1 \mathrm{~s}$ peak at $284.6 \mathrm{eV}$. UV-visible spectra were acquired on a Varian Cary 50 spectrophotometer and recorded using a normal incident angle with respect to the film plane. AFM images were obtained in Tapping and Peak-Force modes using a Multimode 8 microscope equipped with a Nanoscope $\mathrm{V}$ control unit from Bruker operating in ambient air conditions at a scan rate of 0.5-1.2 Hz. To this end, RFESPA-75 (75-100 kHz, and 1.5-6 $\mathrm{N} \mathrm{m}^{-1}$, nominal radius of $8 \mathrm{~nm}$ ) and ScanAsyst-Air-HR (130-160 kHz, and $0.4-0.6 \mathrm{~N} \mathrm{~m}^{-1}$, nominal radius of $2 \mathrm{~nm}$ ) tips, purchased from Bruker, were used. In order to minimize tip convolution effects affecting the AgNPs width, data obtained from AFM image profiling have been corrected according to Canet-Ferrer et al. ${ }^{20}$ Electrical properties of the molecular junctions were recorded with a conductive-AFM (Bruker ICON) under humidity control, ca. $40 \%$, with a $\mathrm{N}_{2}$ flow using the Peak Force Tunnelling AFM $\left(\right.$ PF-TUNA $^{\mathrm{TM}}$ ) mode, and employing a PF-TUNA ${ }^{\mathrm{TM}}$ cantilever from Bruker (coated with $\mathrm{Pt} / \mathrm{Ir} 20 \mathrm{~nm}, c a .25 \mathrm{~nm}$ radius, $0.4 \mathrm{~N} \mathrm{~m}^{-1}$ spring constant and $70 \mathrm{kHz}$ resonance frequency).

Cyclic voltammetry (CV) experiments were performed using a potentiostat from EcoChemie and a standard three electrode cell, where the working electrode was a bare Au(111) electrode, a monolayer modified $\mathrm{Au}(111)$ electrode, or a monolayer/AgNP modified Au(111) electrode. These working electrodes were connected to the potentiostat by means of a cable ended in a metallic tweezer that held the electrode. The reference electrode was $\mathrm{Ag} / \mathrm{AgCl}, \mathrm{KCl}(3 \mathrm{M}$ ) and the counter electrode was a Pt sheet.

\section{Results and discussion}

Langmuir films were formed from $10^{-5} \mathbf{M}$ solutions of $\mathbf{1 H}_{\mathbf{2}}$ in $\mathrm{CHCl}_{3}$ on both pure water and $4 \times 10^{-4} \mathrm{M} \mathrm{AgNO}_{3}$ aqueous subphases. The surface pressure $v$. area per molecule $(\pi-A)$ isotherms obtained for the film from a pure water sub-phase features a lift-off at $c a .0 .80 \mathrm{~nm}^{2}$ molecule ${ }^{-1}$ whilst the monolayer prepared on an $\mathrm{AgNO}_{3}$ aqueous sub-phase, shows the liftoff at a slightly smaller area, ca. $0.65 \mathrm{~nm}^{2}$ molecule ${ }^{-1}$ (Fig. 2). Fig. 2 also includes the surface potential isotherms recorded upon the compression process in both sub-phases. The significantly lower values for the surface potential of Langmuir films on the

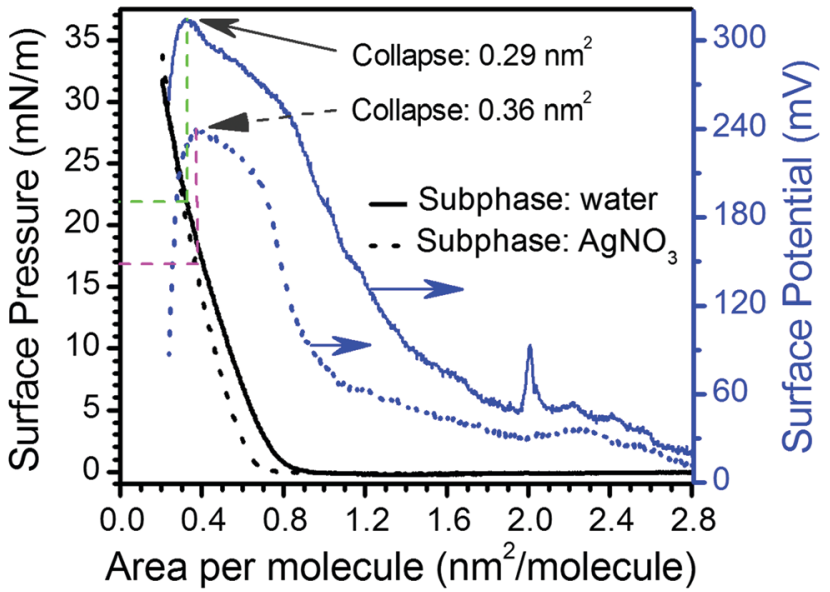

Fig. 2 Representative surface pressure and surface potential vs. area per molecule isotherms formed from $\mathbf{1} \mathbf{H}_{\mathbf{2}}$ on pure water $\left(\mathbf{1 H}_{\mathbf{2}}\right)$ and $4 \times 10^{-4} \mathrm{M}$ $\mathrm{AgNO}_{3}\left(\mathbf{1} \mathrm{HAg}^{+}\right)$aqueous sub-phases.

$\mathrm{AgNO}_{3}$ aqueous sub-phase in comparison to the pure water subphase are indicative of a better charge compensation of the double ionic layer in the presence of the silver salt, which could indicate silver ion complexation by the carboxylate head group. $^{21}$ The surface potential $v s$. area per molecule $(\Delta V-A)$ isotherms clearly evidence the collapse of the monolayers at areas per molecule of $0.29 \mathrm{~nm}^{2}$ (which corresponds to a surface pressure of $22 \mathrm{mN} \mathrm{m}^{-1}$ in the $\pi-A$ isotherm) and $0.36 \mathrm{~nm}^{2}$ (which corresponds to a surface pressure of $17 \mathrm{mN} \mathrm{m}^{-1}$ in the $\pi-A$ isotherm) in water and $\mathrm{AgNO}_{3}$ aqueous sub-phase, respectively (abrupt decrease in the surface potential isotherms). ${ }^{22}$ Brewster angle microscopy (BAM) images confirm the formation of a homogeneous Langmuir film from $\mathbf{1 H}_{2}$ on the $\mathrm{AgNO}_{3}$ aqueous sub-phase, $\mathbf{1 H A g}^{+}$film, without the presence of three-dimensional structures at surface pressures below the collapse of the monolayer (Fig. 3).
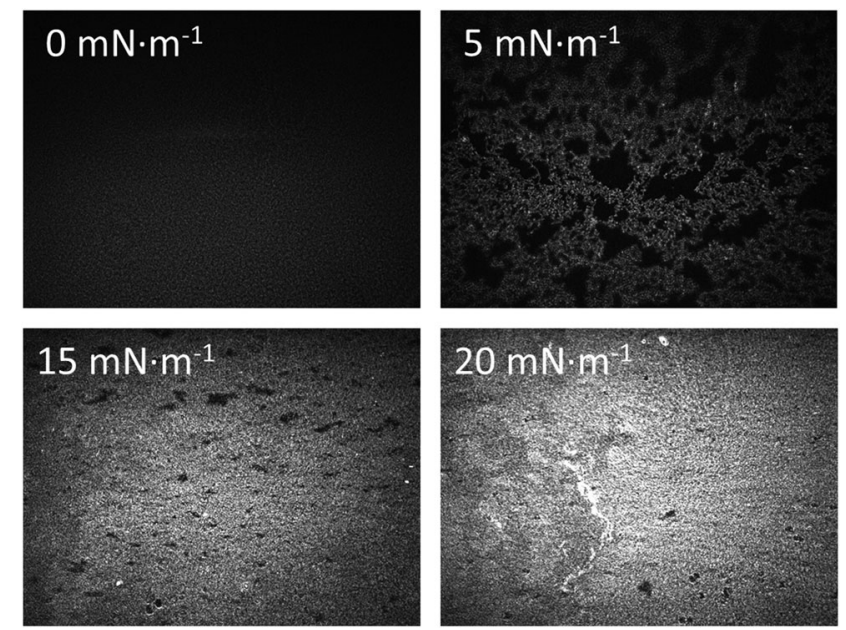

Fig. 3 Brewster Angle Microscopy images of Langmuir films formed from $\mathbf{1 H}_{\mathbf{2}}$ on an $\mathrm{AgNO}_{3}$ aqueous sub-phase $\left(\mathbf{1 H A g}^{+}\right)$at the indicated surface pressures. The collapse of the monolayer can be observed in the bottom right image. The field of view along the $x$ axes for the BAM images is $3300 \mu \mathrm{m}$. 


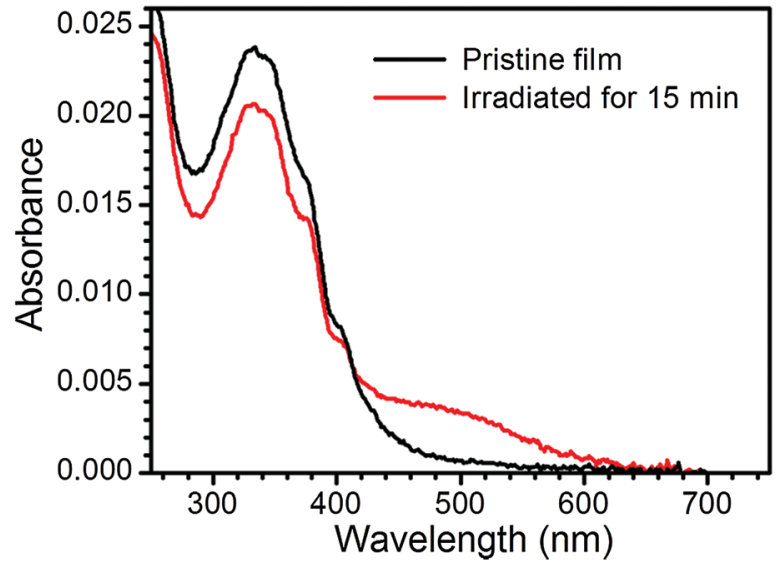

Fig. 4 UV-vis spectra of a pristine single layer $\mathrm{LB}$ film of $\mathbf{A g g}^{+}$and the same film after irradiation with UV light at $254 \mathrm{~nm}$.

The Langmuir monolayers $\mathbf{1 H A g}^{+}$were transferred onto solid substrates, that were initially held outside of the aqueous $\mathrm{AgNO}_{3}$ sub-phase, by the vertical dipping method at a surface pressure of $15 \mathrm{mN} \mathrm{m}^{-1}$ to form monolayer Langmuir-Blodgett films. The transfer ratio (defined as the decrease in monolayer area during the deposition divided by the area of the substrate) calculated using the trough software was 1 . Under these transference conditions (substrates initially outside of the sub-phase) the carboxylic group not immersed in the aqueous sub-phase is directly attached to the substrate, denoted here as $\mathbf{1} \mathbf{A g}^{+}$films. XPS experiments confirm the chemisorption of a carboxylate moiety onto gold substrates, as reported previously. ${ }^{12}$

Fig. 4 shows the UV-vis spectrum of a pristine monolayer LB film of $\mathbf{1} \mathbf{A g}^{+}$transferred onto a quartz substrate from an $\mathrm{AgNO}_{3}$ aqueous solution as sub-phase. This spectrum features a band at $330 \mathrm{~nm}$, which is likely to result from unresolved $\pi-\pi^{*}$ transitions associated with the OPE backbone, ${ }^{23}$ and observed at approximately the same wavelength as the analogous transitions of $\mathbf{1} \mathbf{H}_{2}$ in solution. Irradiation of the LB film $\mathbf{1} \mathbf{A g}^{+}$with UV light $(254 \mathrm{~nm})$ results in the appearance of a small broad peak at ca. $460 \mathrm{~nm}$, attributable to surface plasmon resonance of silver nanoparticles. ${ }^{24}$ The plasmon peak reaches a maximum intensity after 15 minutes of irradiation.

The observation of a plasmon band is consistent with the formation of silver nanoparticles (AgNPs) on top of the LB film after irradiation. These films are denoted 1AgNP to distinguish them from the silver ion complexed films $\mathbf{1 A g}^{+}$. Formation of metallic silver on these monolayers has also been demonstrated by XPS. Fig. 5 shows the XPS spectrum of irradiated LB films of 1AgNP on a gold substrate. The $\mathrm{Ag}(3 \mathrm{~d})$ region for the film after irradiation shows two peaks at 367.8 and $373.8 \mathrm{eV}$ in good agreement with the peaks for $\operatorname{Ag}(0)$ reported in the literature. ${ }^{25,26}$ In addition, the area ratio of $4: 3$ and the peak separation, $6 \mathrm{eV}$, is also consistent with metallic silver. ${ }^{25,26}$

However, neither UV-vis spectroscopy nor XPS provide any information about the distribution of the silver nanoparticles on the surface of the film of 1AgNP. To investigate this issue, the surface was studied by atomic force microscopy (AFM).

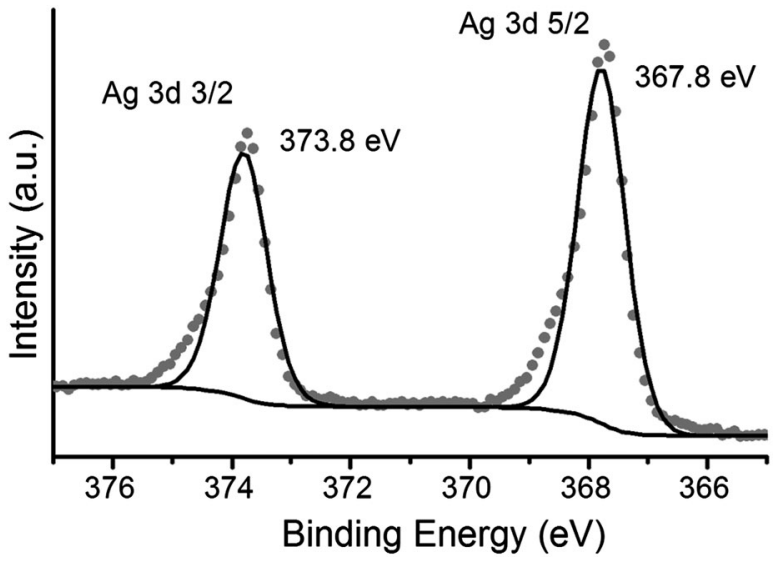

Fig. 5 XPS spectrum of $A g(3 d)$ photoelectrons of a one-layer LB film of 1AgNP, formed following transference of a $\mathrm{LB}$ film of $\mathbf{A g g}^{+}$from an $\mathrm{AgNO}_{3}$ aqueous solution and irradiated at $254 \mathrm{~nm}$ for 15 minutes.

Fig. 6a shows an AFM image of a LB film of $\mathbf{A g g}^{+}$before and after irradiation. In comparison to the smooth and featureless surface exhibited by a pristine LB film of $\mathbf{1} \mathbf{A g}^{+}$(surface roughness, calculated in terms of the Root Mean Square (RMS), $0.4 \pm 0.1 \mathrm{~nm}$ over areas of $300 \times 300 \mathrm{~nm}^{2}$ ), after irradiation an organic-layer modified substrate homogeneously covered by disk-shaped particles closely assembled into a tightly packed 2D-arrangement, with low occurrence of irregular 3D Ag-aggregates is obtained. The RMS roughness of irradiated-film is $2.6 \pm 0.2 \mathrm{~nm}$, clearly much greater than that of the original $\mathbf{1} \mathbf{A g}^{+}$film. A statistical analysis of the AFM images reveal that these AgNPs have an average diameter of around $28 \mathrm{~nm}$ (corrected by the tip convolution) and an average height of $c a .6 .9 \mathrm{~nm}$ (Fig. $6 \mathrm{~b}$ and c). Additionally, AFM images indicate a large surface coverage by the silver nanoparticles. A bearing analysis of the AFM images was made. In a bearing analysis, the depths of all pixels of the image with respect to a reference point, i.e., the highest pixel are analysed. This kind of analysis renders an accurate estimation of the percentage of area covered by features, i.e. surface coverage, at every pixel depth. ${ }^{27,28}$ The bearing analysis of a one-layer LB film of 1AgNP gave an estimated surface coverage of $76 \%$ (see Fig. 7). This surface coverage value is significantly higher than that observed for the photoreduction of a gold precursor, ${ }^{11}$ which exhibited a surface coverage of $25 \%$, indicating that silver has a larger tendency to form extended structures across the LB film. This result represents a step forward since this large surface packing of the silver nanoparticles may facilitate a subsequent step towards the complete metallization of monolayers by chemical vapour or electroless deposition processes without damaging the underlying organic monolayer.

As noted above, a frequent difficulty encountered in the fabrication of metal-monolayer-metal devices is the deposition of the top contact electrode without the formation of shortcircuits as a consequence of penetration of the growing topcontact electrode through the monolayer and subsequent contact with the underlying bottom electrode. ${ }^{29-31}$ Consequently, it is critical to verify whether the irradiation of an LB film of $\mathbf{1 A g}^{+}$to generate $\mathbf{1 A g N P}$ leads to short-circuits or if the layer-like 
(a)

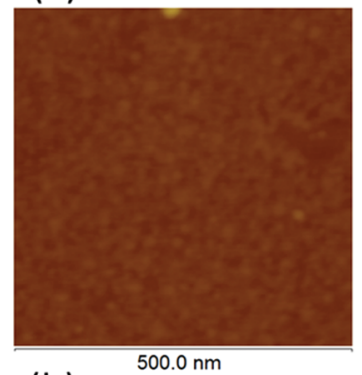

(b)

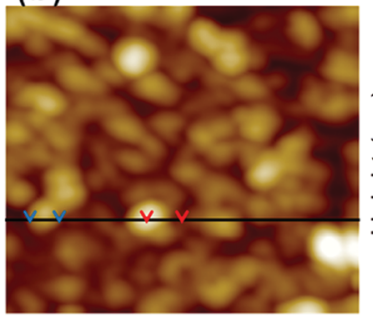

(c)
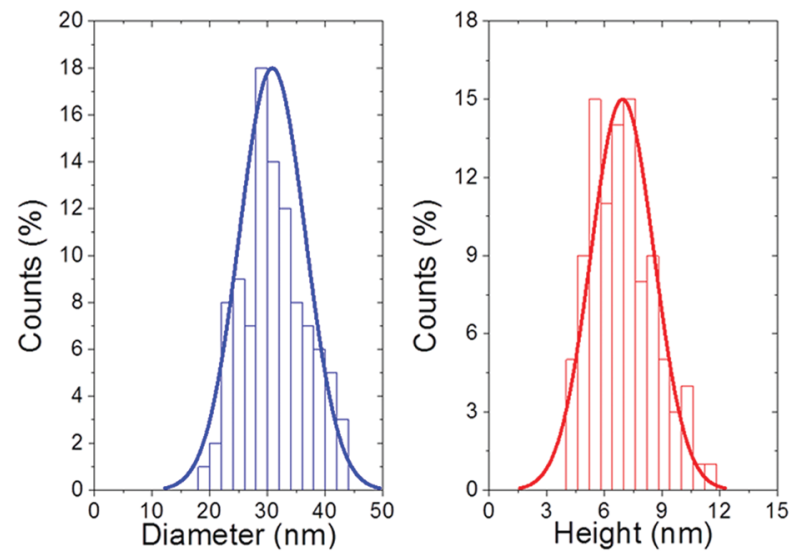

$<\mathrm{d}>=(28.6 \pm 5.7) \mathrm{nm}$

$<\mathrm{h}\rangle=(6.9 \pm 1.6) \mathrm{nm}$

Fig. 6 (a) $500 \times 500 \mathrm{~nm}^{2}$ AFM images of a monolayer LB film of $\mathbf{1} \mathbf{A g}^{+}$ transferred from an $\mathrm{AgNO}_{3}$ aqueous solution before (left panel) and after irradiation for 15 minutes at $254 \mathrm{~nm}$ (right panel). (b) Cross section of a representative AFM image and analysis profile illustrating the dimensions of the AgNPs. (c) Histograms showing the particle diameter (blue line) and height distribution (red line) corresponding to 100 AgNPs taken from different AFM images. Averaged NPs diameter and height values are depicted in the box below.

arrangement of AgNPs formed from this soft photochemical method is an effective route towards a top-contact electrode. For that, $I-V$ curves were recorded for these metal-monolayerAgNPs structures using a conductive-AFM, c-AFM. ${ }^{32-36}$ The AFM system is equipped with a low noise current amplifier and $\mathrm{Pt} / \mathrm{Ir}$ coated AFM tips were used, with a typical elastic contact value of around $0.5 \mathrm{~N} \mathrm{~m}^{-1}$ (PFTUNA, Bruker). Images were taken using the peak-force tapping mode, in which the tip makes intermittent contact with the surface at a frequency of $2 \mathrm{kHz}$. The maximum force (peak-force) is set typically below $10 \mathrm{nN}$, to limit damage to the surface and detrimental lateral forces.
These characteristics make the peak-force tapping mode a useful strategy for the conductivity mapping of soft or fragile samples, since lateral forces are largely avoided. After acquiring. an image, $I-V$ curves were recorded by positioning the AFM tip on a specific location of the surface (for example, on top of an AgNP), establishing contact at a suitable force (usually larger than the peak-force value) and applying a bias between the LB-coated gold substrate and the tip. Too much force results in unacceptably large deformation of the monolayer underlying the AgNPs, while too little force yields an inadequate electrical contact between the AFM probe tip and the AgNP. Fig. 8 shows how an increase in the applied force results in a more effective contact between the tip and the AgNPs leading to a higher conductance. It is worth indicating here that these high forces $(17.5$ or $24 \mathrm{nN})$, required to make a reasonable contact, do not damage the organic layer during the determination of the electrical properties. Fig. 9c shows a representative $I-V$ of all the curves ( $c a .250$ curves) recorded using a set-point force of $17.5 \mathrm{nN}$ whilst Fig. 10 shows the conductance histogram built by adding all the experimental data in the -0.5 to $0.5 \mathrm{~V}$ ohmic region for each of the $250 \mathrm{I}-\mathrm{V}$ curves obtained experimentally at a set-point force of $17.5 \mathrm{nN}$. These $I-V$ curves exhibit a linear section only at relatively low bias voltages and increasing curvature at higher bias, which is the common behaviour observed in metal-molecule-metal junctions. Importantly, no low resistance trace characteristics of metallic short circuits have been observed. In addition, Fig. 9a and b show a representative $I-V$ curve registered for an $\mathrm{LB}$ film of $\mathbf{1} \mathbf{H}_{\mathbf{2}}$ prepared onto a $0.1 \mathrm{M} \mathrm{NaOH}$ subphase and an $I-V$ curve recorded on regions of the 1AgNP film not covered by AgNPs. These curves also exhibit the typical shape observed for metal-moleculemetal junctions, which rules out the presence of short-circuits and confirms that robust and reliable top-contacts have been prepared by photoreduction of a silver precursor without damaging the underlying organic monolayer film or altering/ contaminating the interfaces.

In order to reinforce the conclusion that there are no metallic short circuits, a transition voltage spectroscopy (TVS) analysis has been made (Fig. 10b). ${ }^{37}$ It is known that the charge transport mechanism in electronic junctions of conjugated molecules undergoes a transition from direct tunnelling (rectangular barrier) to field emission (triangular barrier) upon increasing the applied bias. The voltage at which this transition occurs $\left(V_{\text {trans }}\right)$ is linearly correlated with the energy offset between the metal Fermi level and the highest occupied molecular orbital (HOMO). ${ }^{37}$ The obtained $V_{\text {trans }}$ from the TVS analysis, $0.84 \pm$ $0.07 \mathrm{~V}$, is in excellent agreement other similar OPE derivatives, ${ }^{38}$ which also rules out the presence of short circuits since short circuits would result in low values of $V_{\text {trans. }}$.

Cyclic voltammetry (CV) experiments have been used as a further confirmation that no significant alterations of the organic monolayer took place during the photoreduction process. Thus, CV experiments using working electrodes modified by the three different steps associated with the fabrication of the $\mathrm{Au}(111) /$ monolayer of 1AgNP devices were recorded in $0.1 \mathrm{M} \mathrm{NaOH}$ and the obtained results are presented in Fig. 11. 

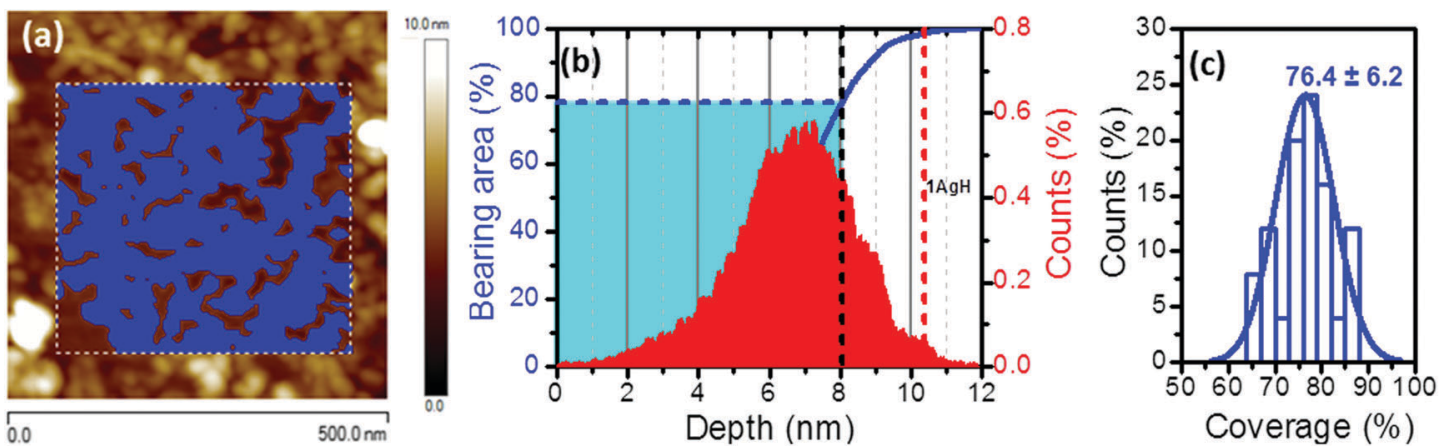

Fig. 7 (a) $500 \times 500 \mathrm{~nm}^{2}$ AFM images of a monolayer LB film of 1 AgNP transferred from an $\mathrm{AgNO}_{3}$ aqueous solution and after irradiation for 15 minutes at $254 \mathrm{~nm}$ with the mask in blue unveiling nanoparticle-free molecule-modified areas. (b) Depth histogram showing the distribution of height data at different depth referred to a reference point, i.e. the highest pixel. The blue line (bearing analysis) indicates the relative projected area covered at each depth value depicted as a blue mask in the topographic image corresponding to the white-dashed boxed area. The small peak in the histogram marked with a red-dashed vertical line is attributed to the nanoparticle-free molecule-modified substrate while the black line accounts for the selected height threshold corresponding to half of the averaged height of the measured nanoparticles. (c) Histogram showing the average percentage of AgNPs-covered substrate area obtained for fifty equivalent but different $500 \times 500 \mathrm{~nm}^{2}$ AFM images.

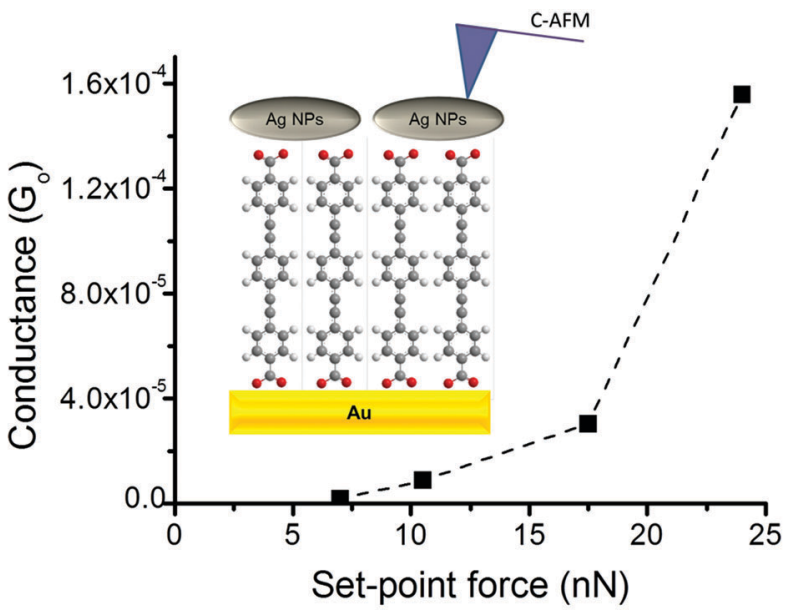

Fig. 8 Average conductance values measured by locating the tip of the C-AFM on top of AgNPs at the indicated set-point forces. Inset: A scheme of the studied metal|1AgNPs structures.

The electrochemical response recorded for the bare gold electrode corresponds well with that typically observed for $\mathrm{Au}(111)$-oriented surfaces in alkaline media. ${ }^{39}$ In particular characteristic voltammetric peaks related to the gold oxide formation (nominally described here as AuO) are observed, identified with black letters as A1 and A2, as well as the corresponding electroreduction in the cathodic scan, labelled as C. Once the $\mathrm{Au}(111)$ electrode is modified with a single layer LB film, a dramatic decrease in the charge density along with a shift towards more positive potentials is observed for the electrochemical formation of the gold oxide monolayer. This corresponds to an inhibition of $\mathrm{AuO}$ formation since the surface is initially covered with a single layer LB film. At the most positive potentials $(>0.5 \mathrm{~V})$ current is seen to flow which could correspond to $\mathrm{AuO}$ formation and perhaps partial oxidation of the organic monolayer film. Since a peak is seen at the potential expected for $\mathrm{AuO}$ reduction in the reverse sweep it is reasonable to assume that the anodic peak at $E>0.5 \mathrm{~V}$ corresponds mainly to oxide formation on the gold surface beneath the organic monolayer. Finally, the electrochemical response of the AgNPs deposited onto the monolayer, resembles closely that previously reported for AgNP-based electrodes in alkaline media, ${ }^{40}$ since it exhibits two anodic and two cathodic voltammetric peaks marked (in blue) as $\mathrm{A}^{\prime} 1, \mathrm{~A}^{\prime} 2$, and $\mathrm{C}^{\prime} 1, \mathrm{C}^{\prime} 3$, as well as a poorly resolved feature at $\mathrm{C}^{\prime} 2$, respectively. Although the stoichiometry
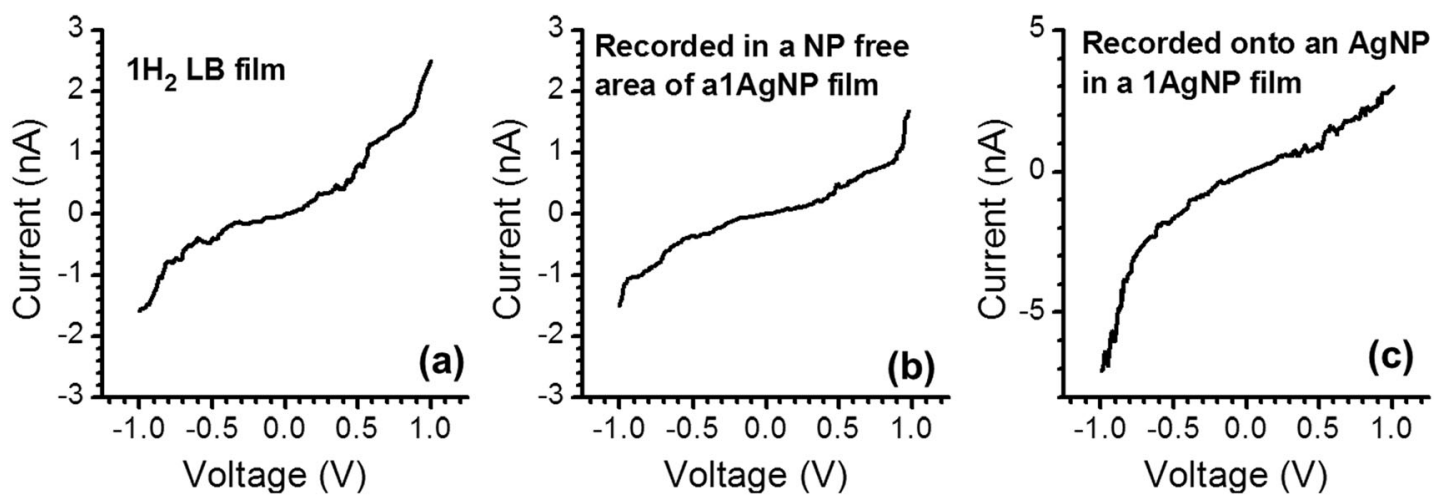

Fig. 9 Representative $I-V$ curves for the indicated films. The set-point force used was $8 \mathrm{nN}$ for (a) and (b) and $17.5 \mathrm{nN}$ for (c). 

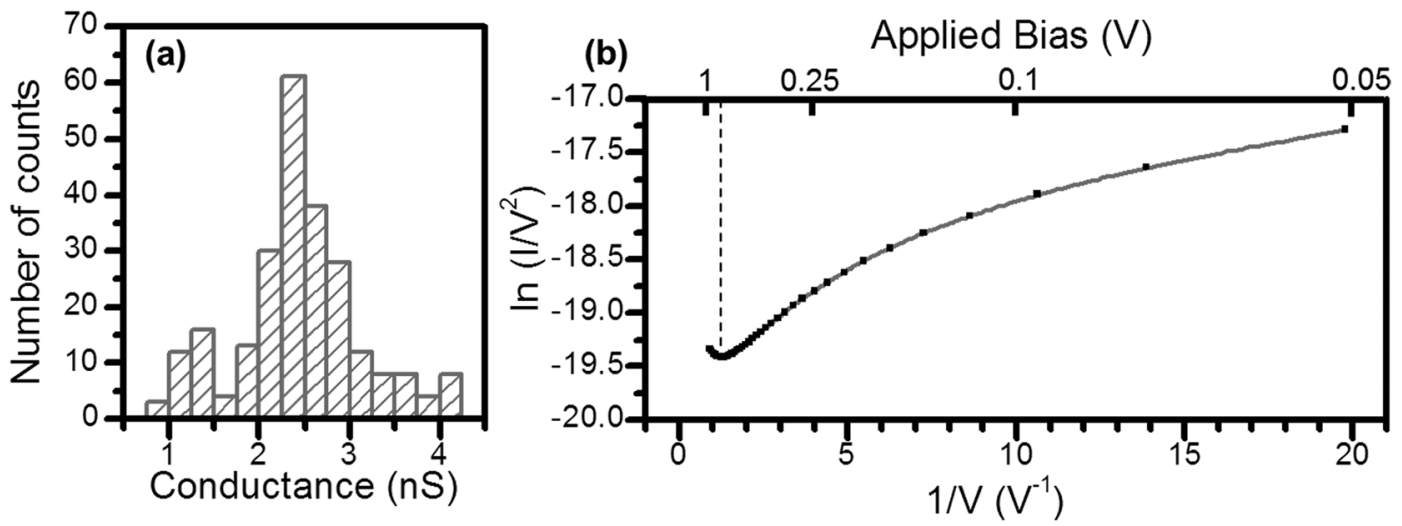

Fig. 10 (a) Conductance histogram built from all the experimental data from -0.5 to $0.5 \mathrm{~V}$ for each $/-V$ curve recorded (ca. 250 curves) positioning the tip onto an AgNP and applying a set point force of $17.5 \mathrm{nN}$. (b) The solid squares represent the average of $10 I-V$ curves for a $1 \mathrm{AgNP}$ device. The dashed vertical line corresponds to the voltage at which the tunnelling barrier transition occurs $\left(V_{\text {trans }}\right)$.

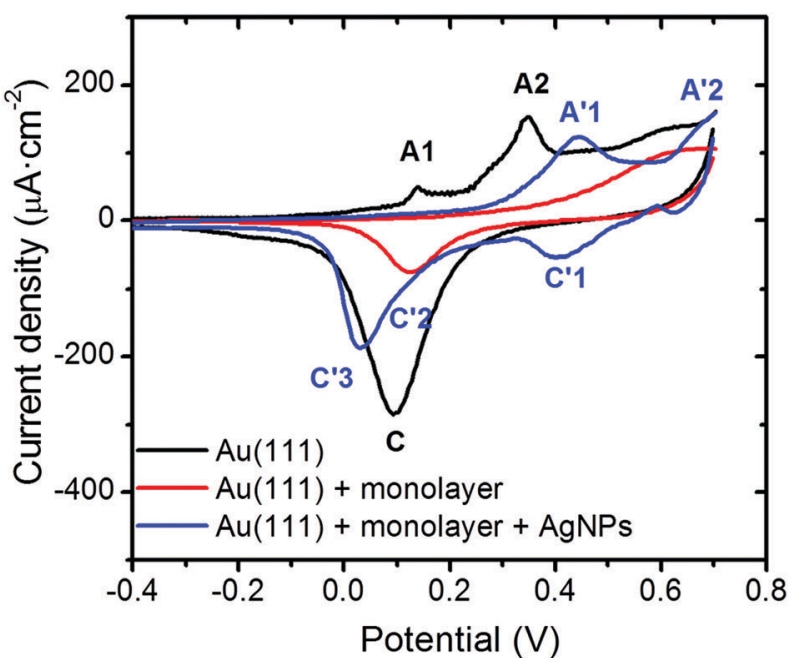

Fig. 11 Black: cyclic voltammograms recorded for a bare Au(111) electrode. Red: monolayer of $\mathbf{1 H}_{\mathbf{2}}$ transferred onto a $\mathrm{Au}(111)$ substrate. Blue: $\mathrm{Au}(111) /$ monolayer with overlying silver nanoparticles (1AgNP). All the voltammograms were recorded in a $0.1 \mathrm{M} \mathrm{NaOH}$ aqueous solution at $0.1 \mathrm{~V} \mathrm{~s}^{-1}$ using a $\mathrm{Ag} / \mathrm{AgCl}, \mathrm{KCl}(3 \mathrm{M})$ reference electrode.

of the formed surface oxides are not well characterized for such conditions, based on the chemistry of silver these could correspond successively to the formation (A) and reduction (C) of the $\mathrm{Ag}(\mathrm{OH})_{2}$ and $\mathrm{Ag}_{2} \mathrm{O}$ monolayer, $\mathrm{Ag}_{2} \mathrm{O}$ multilayer, and finally the oxidation of $\mathrm{Ag}_{2} \mathrm{O}$ to $\mathrm{AgO}$. Note that for this system the exposed silver surface area from the nanoparticles decorating the surface will be much larger than the smooth $\mathrm{Au}(111)$ substrate and as such the contributions from the formation of oxide on the underlying $\mathrm{Au}(111)$ surface are negligible compared to oxidation of the AgNPs in this voltammogram. Therefore, after the formation of AgNPs on the external surface of the single layer LB film (1AgNP), electron transfer through the organic layer occurs and, consequently, the applied electrochemical potential is experienced by the outer AgNPs/electrolyte interface as elegantly stated by Allongue et $a l^{41}$ and supported by Gooding and co-workers. ${ }^{42}$ These results further confirm that the photoreduction of silver cations to metallic nanoparticles results in a robust sandwiched composite comprising a gold singlecrystal, a tightly packed and almost defect free 2D-organic monolayer, and a silver-nanoparticle-based top contact.

\section{Conclusions}

In this contribution, photoreduction of a silver cation coordinated to a LB film terminated in a carboxylic group is shown to be suitable for the fabrication of a top-contact metal electrode in molecular junctions with a large surface coverage. Additionally, it has been shown that this method does not result in short-circuits which is a rather common problem in other traditional techniques for the preparation of top contact electrodes. The large surface coverage achieved would facilitate the subsequent application of other methods to achieve a complete metallization of the monolayer minimizing the risk of short circuits (e.g., electroless deposition, metal evaporation, etc.). Also the use of masks that allow the irradiation of the desired areas of the sample would result in the fabrication of arrays of devices.

\section{Acknowledgements}

S. M. F. P.-M and P. C. are grateful for financial assistance from Ministerio de Economía y Competitividad from Spain and fondos FEDER in the framework of projects CTQ2012-33198, CTQ2013-50187-EXP, CSIC10-4E-805, and CSD2010-00024. S. M. and P. C. also acknowledge DGA and fondos FEDER for funding the research group Platón (E-54). R. J. N., P. J. L. and S. M.-G. thank EPSRC for funding (EPSRC Grants EP/K007785/1, EP/H035184/1, and EP/K007548/1). P. J. L. holds an ARC Future Fellowship (FT120100073) and gratefully acknowledges funding for this work from the ARC (DP140100855).

\section{References}

1 Editorial, Nat. Nanotechnol., 2013, 8, 385.

2 D. Xiang, X. Wang, C. Jia, T. Lee and X. Guo, Chem. Rev., 2016, 4318. 
3 H. Haick and D. Cahen, Prog. Surf. Sci., 2008, 83, 217.

4 D. Vuillaume, C. R. Phys., 2008, 9, 78.

5 H. B. Akkerman and B. de Boer, J. Phys.: Condens. Matter, 2008, 20, 013001.

6 D. Vuillaume, Proc. IEEE, 2010, 98, 2111.

7 A. V. Walker, J. Vac. Sci. Technol., A, 2013, 31, 050816.

8 P. Cea, L. M. Ballesteros and S. Martin, Nanofabrication, 2014, 1, 96.

9 L. M. Ballesteros, S. Martin, J. Cortés, S. Marqués-Gonzalez, F. Pérez-Murano, R. J. Nichols, P. J. Low and P. Cea, Adv. Mater. Interfaces, 2014, 1, 1400128.

10 H. M. Osorio, P. Cea, L. M. Ballesteros, I. Gascon, S. MarquésGonzález, R. J. Nichols, F. Pérez-Murano, P. J. Low and S. Martín, J. Mater. Chem. C, 2014, 2, 7348.

11 S. Martin, G. Pera, L. M. Ballesteros, A. J. Hope, S. MarquésGonzález, P. J. Low, F. Perez-Murano, R. J. Nichols and P. Cea, Chem. - Eur. J., 2014, 20, 3421.

12 L. M. Ballesteros, S. Martín, J. Cortés, S. Marqués-González, S. J. Higgins, R. J. Nichols, P. J. Low and P. Cea, Chem. - Eur. J., 2013, 19, 5352.

13 I. Person, Pure Appl. Chem., 2010, 82, 1901.

14 N. E. Schlotter, M. D. Porter, T. B. Bright and D. L. Allara, Chem. Phys. Lett., 1986, 132, 93.

15 S. A. Jadhav, Cent. Eur. J. Chem., 2011, 9, 369.

16 H. Hada, Y. Yonezawa, A. Yoshida and A. Kurakake, J. Phys. Chem., 1976, 80, 2728.

17 M. Harada and E. Katagiri, Langmuir, 2010, 26, 17896.

18 Y. Battie, N. Destouches, L. Bois, F. Chassagneux, A. Tishchenko, S. Parola and A. Boukenter, J. Phys. Chem. C, 2010, 114, 8679.

19 W. Haiss, D. Lackey and J. K. Sass, J. Chem. Phys., 1991, 95, 2193.

20 J. Canet-Ferrer, E. Coronado, A. Forment-Aliaga and E. Pinilla-Cienfuegos, Nanotechnology, 2014, 25, 395703.

21 P. Cea, S. Martín, A. Villares, D. Möbius and M. C. López, J. Phys. Chem. B, 2006, 110, 963.

22 G. Pera, A. Villares, M. C. Lopez, P. Cea, D. P. Lydon and P. J. Low, Chem. Mater., 2007, 19, 857.

23 A. Beeby, K. Findlay, P. J. Low and T. B. Marder, J. Am. Chem. Soc., 2002, 124, 8280.
24 S. Pal, Y. K. Tak and J. M. Song, Appl. Environ. Microbiol., 2007, 73, 172.

25 B. de Boer, M. M. Frank, Y. J. Chabal, W. Jiang, E. L. Garfunkel and Z. Bao, Langmuir, 2004, 20, 1539.

26 R. G. Nuzzo and D. L. Allara, J. Am. Chem. Soc., 1983, 105, 4481.

27 S. Y. Heriot, H.-L. Zhang, S. D. Evans and T. H. Richardson, Colloids and Surfaces A: Physicochemical and Engineering Aspects, 2006, 278, 98.

28 M. Haro, B. Giner, C. Lafuente, M. C. Lopez, F. M. Royo and P. Cea, Langmuir, 2005, 21, 2796.

29 A. C. Dürr, F. Schreiber, M. Kelsch, H. D. Carstanjen and H. Dosch, Adv. Mater., 2002, 14, 961.

30 A. V. Walker, T. B. Tighe, J. Stapleton, B. C. Haynie, S. Upilli, D. L. Allara and N. Winograd, Appl. Phys. Lett., 2004, 84, 4008.

31 C. Silien and M. Buck, J. Phys. Chem. C, 2008, 112, 3881.

32 B. Pittenger, N. Erina and D. Su, Application Note Veeco Instruments Inc, 2010.

33 T. J. Young, M. A. Monglus, T. L. Burnett, W. R. Broughton, S. L. Ogin and P. A. Smith, Meas. Sci. Technol., 2011, 22, 125703.

34 K. Sweers, K. van der Werf, M. Bennink and V. Bubramaniam, Nanoscale Res. Lett., 2011, 6, 270.

35 G. Lee, H. Lee, K. Nam, J.-H. Han, J. Yang, S. W. Lee, D. S. Yoon, K. Eom and T. Kwon, Nanoscale Res. Lett., 2012, 7, 608.

36 M. Lorenzoni, L. Evangelio, S. Verhaeghe, C. Nicolet, C. Navarro and F. Pérez-Murano, Langmuir, 2015, 42, 11630.

37 J. M. Beebe, B. Kim, J. W. Gadzuk, C. D. Frisbie and J. G. Kushmerick, Phys. Rev. Lett., 2006, 97, 026801.

38 J. M. Beebe, B. Kim, C. D. Frisbie and J. G. Kushmerick, ACS Nano, 2008, 2, 827.

39 P. Rodríguez, N. Garcia-Araez and M. T. M. Koper, Phys. Chem. Chem. Phys., 2010, 12, 9373.

40 A. C. Joshi, G. B. Markad and S. K. Haram, Electrochim. Acta, 2015, 161, 108.

41 J.-N. Chazalviel and P. Allongue, J. Am. Chem. Soc., 2011, 133, 762.

42 A. Barfidokht, S. Ciampi, E. Luais, N. Darwish and J. J. Gooding, Anal. Chem., 2013, 85, 1073. 